\title{
Topic Enhanced Controllable CVAE for Dialogue Generation (Student Abstract)
}

\author{
Yiru Wang, ${ }^{1}$ Pengda $\mathrm{Si},{ }^{1}$ Zeyang Lei, ${ }^{* 2}$ Yujiu Yang ${ }^{\dagger 1}$ \\ ${ }^{1}$ Tsinghua Shenzhen International Graduate School, Tsinghua University, P. R. China \\ ${ }^{2}$ Baidu Inc., Beijing, P. R. China \\ \{wangyiru17, spd18\}@mails.tsinghua.edu.cn, zeyanglei@gmail.com, yang.yujiu@sz.tsinghua.edu.cn
}

\begin{abstract}
Neural generation models have shown great potential in conversation generation recently. However, these methods tend to generate uninformative or irrelevant responses. In this paper, we present a novel topic-enhanced controllable CVAE (TECCVAE) model to address this issue. On the one hand, the model learns the context-interactive topic knowledge through a novel multi-hop hybrid attention in the encoder. On the other hand, we design a topic-aware controllable decoder to constrain the expression of the stochastic latent variable in the CVAE to reduce irrelevant responses. Experimental results on two public datasets show that the two mechanisms synchronize to improve both relevance and diversity, and the proposed model outperforms other competitive methods.
\end{abstract}

\section{Introduction}

Neural dialogue systems tend to generate monotonous and meaningless responses due to the information loss in the encoding phase, and the information provided only by the encoding vector obtained from the encoder for the decoding phase is far from sufficient. Most existing works introduce additional information such as context (Serban et al. 2016), keyword (Xing et al. 2017) or knowledge-base (Young et al. 2018) to improve response generation quality. Conditional variational auto-encoders (CVAEs) (Zhao, Zhao, and Eskénazi 2017) can generate informative responses relying on the variability of the stochastic latent variable. Although effective, many methods establish a trade-off between relevance and diversity. For example, in CVAE, the randomness of the latent variable can result in responses deviated from the conversation context. Traditional keyword methods are limited by the vocabulary size and the fixed keyword number, also not able to dynamically interact with the context information to learn the comprehensive topic knowledge.

To address the aforementioned limitation, we propose a novel topic enhanced controllable CVAE (TEC-CVAE)

\footnotetext{
${ }^{*}$ This work was done when Zeyang Lei was in Tsinghua Univ.

${ }^{\dagger}$ Corresponding author. This work was supported partially by Guangdong Basic and Applied Basic Research Foundation (No.2019A1515011387), and Shenzhen Basic Research Foundation (No.JCYJ201704is170118573).

Copyright (C) 2020, Association for the Advancement of Artificial Intelligence (www.aaai.org). All rights reserved.
}

model, which aims to leverage the context-interactive topic knowledge and topic-aware controllable latent variable to further improve the relevance and informativeness of generated responses. More specifically, (1) in the encoder, we introduce a coarse keyword sequence with additional multihop hybrid attention layers. The interrelationship among keywords is learnt to fuse the topic information throughout the dialogue for each keyword representation. At the same time, a specific contextual topic vector is produced based on the interaction between the utterance and the keyword vectors above for final representation of each utterance. (2) In the decoder, we utilize an overall topic vector to generate the latent variable. Furthermore, we design a novel expression gate to dynamically control the influence of the randomness brought by the latent variable to avoid generating irrelevant responses. The experimental results show that our model is efficient and achieves state-of-the-art results.

\section{Our Model}

We extract nouns and named entities (NE) in the input dialogue histories as topic keywords. If no nouns and NE, we use adjectives and verbs instead. We then filter universal words according to mutual information, and finally get the keyword sequence $K=\left[k_{1}, \ldots, k_{m}\right]$. The encoder is a hierarchical network with sentence-level and context-level layers. The sentence-level encoder produces an encoding vector for each utterance, and obtains the sequence of utterance representations $U=\left[u_{1}, \ldots, u_{n}\right]$ for input dialogue histories.

\section{Multi-hop Hybrid Attention}

We first apply a self-attention layer on the keyword sequence $K$ to obtain the representation $r_{i}$ for $k_{i}$. Then, we calculate the attention of the utterance vector $u_{i}$ to the keyword vectors to produce a contextual fused topic vector $t_{i}$ for each utterance.

$$
\begin{aligned}
r_{i} & =\sum_{j=1}^{m} a_{i j}^{k} e_{j}, \text { with } a_{i j}^{k}=\frac{\exp \left(\sigma\left(\left[e_{i}, e_{j}\right]\right)\right.}{\sum_{p=1}^{m} \exp \left(\sigma\left(\left[e_{i}, e_{p}\right)\right]\right.} \\
t_{i} & =\sum_{j=1}^{m} a_{i j}^{u} r_{j}, \text { with } a_{i j}^{u}=\frac{\exp \left(\sigma\left(\left[u_{i}, r_{j}\right]\right)\right.}{\sum_{p=1}^{m} \exp \left(\sigma\left(\left[u_{i}, r_{p}\right)\right]\right.}
\end{aligned}
$$


where $e_{i}$ is the word embedding of the keyword $k_{i} . \sigma$ is a score function that defines the relation between two vectors as $\sigma\left(\left[u_{i}, r_{j}\right]\right)=u_{i} \cdot\left(w r_{j}\right)^{T} / \sqrt{d_{u}}$, where $d_{u}$ is the dimension of $u_{i}$, and $w$ is the parameter vector to be learnt.

In this way, each keyword representation actually fuses the global coarse dialogue information. Furthermore, we concatenate $u_{i}$ and $t_{i}$ as the final representation $u_{i}^{h}=\left[u_{i} ; t_{i}\right]$ of the $i$-th utterance, which compresses the context information into $u_{i}^{h}$ via $t_{i}$. The context-level encoder receives the sequence $\left[u_{1}^{h}, \ldots, u_{n}^{h}\right]$ to generate the context vector $c$.

\section{Topic-aware Controllable Decoder}

We introduce the overall topic vector $t^{o}$ by applying a meanpooling on the keyword representations $t^{o}=\sum_{i=1}^{m} r_{i} / m$ into the latent variable $z$ generation in CVAE. Specifically, the recognition network $q_{\phi}\left(z \mid y, c, t^{o}\right) \sim \mathcal{N}\left(\mu, \sigma^{2} I\right)$ is with $\left[\mu ; \log \left(\sigma^{2}\right)\right] \triangleq W_{r}\left[y ; c ; t^{o}\right]+b_{r}$, where $y$ is the encoding vector of the target response. The prior network $p_{\theta}\left(z \mid c, t^{o}\right) \sim \mathcal{N}\left(\mu^{\prime}, \sigma^{\prime 2} I\right)$ is with $\left[\mu^{\prime} ; \log \left(\sigma^{\prime 2}\right)\right] \triangleq$ $\operatorname{MLP}\left(\left[c ; t^{\circ}\right]\right)$. The decoder is a GRU network with initial state $s_{0}=W_{i}\left[z ; c ; t^{o}\right]+b_{i}$. For word prediction, we design an expression gate $g^{e}$ in the GRU cell to dynamically control the strength of $z$. The decoding at time step $t$ is as follows:

$$
\begin{aligned}
g_{t}^{e} & =\sigma\left(W_{x}^{e}\left[y_{t} ; t^{o}\right]+W_{h}^{e} s_{t-1}+W_{z}^{e} z\right) \\
\tilde{s_{t}} & =f\left(W_{x}\left[y_{t} ; t^{o}\right]+W_{h}\left(g_{t}^{r} \circ s_{t-1}\right)+W_{z}\left(g_{t}^{e} \circ z\right)\right) \\
s_{t} & =\left(1-g_{t}^{z}\right) \circ s_{t-1}+g_{t}^{z} \circ \tilde{s_{t}}
\end{aligned}
$$

where $g^{r}$ and $g^{z}$ are the reset gate and update gate of GRU. $y_{t}$ is the $t$-th input word. $\sigma$ is the sigmoid function and $f$ is the tanh function. We allow the dynamically controllable $z$ to participate in the generation at each decoding step.

\section{Experiments}

\section{Datasets}

All the experiments in this paper are carried out on two public datasets, one is the DailyDialog dataset collected by $\mathrm{Li}$ et al. (2017) and the other is the OpenSubtitles dataset from the OpenSubtitles website ${ }^{1}$.

\section{Baseline Methods}

We compare our model with several state-of-the-art baseline methods, including the attention-based sequence-tosequence model(S2SA) (Bahdanau, Cho, and Bengio 2015), HRED (Serban et al. 2016) and CVAE (Zhao, Zhao, and Eskénazi 2017).

\section{Implementation Details}

In all experiments, all the initial weights are sampled from a uniform distribution $[-0.08,0.08]$ and all the bias vectors are initialized to zero. The word embedding size is 200 and the topic vector size is 30 . The sentence encoder, context encoder and response decoder are GRU with hidden size of 300, 600 and 400 respectively. The $M L P$ in the prior network has a hidden size of 400 , and the latent variable $z$ has a size of 200. We use the Adam optimizer with batch size 30 . The learning rate is 0.001 and the gradient clip is at 5 .

\begin{tabular}{|c|c|c|c|c|}
\hline & \multicolumn{5}{|c|}{ Dailydialog Corpus } \\
\hline Method & BLEU & Dist-1 & Dist-2 & KL cost \\
\hline S2SA & 10.30 & $884 / 0.0134$ & $2382 / 0.0495$ & - \\
\hline HRED & 11.36 & $872 / 0.0123$ & $2399 / 0.0470$ & - \\
\hline CVAE & 14.99 & $2766 / 0.0269$ & $14791 / 0.1876$ & 17.32 \\
\hline MHA-CVAE & 15.38 & $3005 / \mathbf{0 . 0 2 9 5}$ & $15879 / 0.2033$ & 17.44 \\
\hline TACD-CVAE & 15.63 & $3002 / 0.0275$ & $16963 / 0.2035$ & 18.02 \\
\hline TEC-CVAE & $\mathbf{1 6 . 1 5}$ & $\mathbf{3 2 2 8} / 0.0279$ & $\mathbf{1 8 1 1 1 / 0 . 2 0 4 0}$ & $\mathbf{1 8 . 4 1}$ \\
\hline & \multicolumn{5}{|c|}{ OpenSubtitles Corpus } \\
\hline S2SA & 7.53 & $271 / 0.0031$ & $648 / 0.0136$ & - \\
\hline HRED & 10.46 & $325 / 0.0033$ & $704 / 0.0122$ & - \\
\hline CVAE & 12.16 & $3063 / 0.0228$ & $20972 / 0.2242$ & 14.19 \\
\hline MHA-CVAE & 12.27 & $3461 / 0.0256$ & $21446 / 0.2266$ & 14.70 \\
\hline TACD-CVAE & $\mathbf{1 2 . 5 9}$ & $4172 / 0.0297$ & $24901 / 0.2491$ & 15.04 \\
\hline TEC-CVAE & 12.41 & $\mathbf{4 3 6 5 / 0 . 0 3 1 5}$ & $\mathbf{2 5 7 2 8 / 0 . 2 6 1 8}$ & $\mathbf{1 6 . 0 9}$ \\
\hline
\end{tabular}

Table 1: Evaluation results. MHA-CVAE and TACD-CVAE represent that only multi-hop hybird attention or topic-aware controllable decoder is used in our model, respectively.

\section{Experimental Results}

We adopt BLEU and Dist- $n$ to evaluate the relevance and diversity respectively. Dist- $n$ are the number and proportion of distinct $n$-grams in all generated tokens. The experimental results are shown in Table 1. Compared to the baseline methods, our models achieve better results on both datasets. Higher KL cost indicates our model learns a more meaningful latent variable which benefits from the integrated topic knowledge. Two variant models are superior to baselines and TEC-CVAE gains the best overall performance. This verifies that both mechanisms contribute to the improvement of diversity and relevance simultaneously in response generation.

\section{Conclusion}

We propose a novel topic enhanced controllable CVAE model which learns the context-interactive topic knowledge and topic-aware controllable latent variable. Experimental results show that our method achieves better results in both relevance and informativeness of generated responses.

\section{References}

Bahdanau, D.; Cho, K.; and Bengio, Y. 2015. Neural machine translation by jointly learning to align and translate. ICLR abs/1409.0473.

Li, Y.; Su, H.; Shen, X.; Li, W.; Cao, Z.; and Niu, S. 2017. Dailydialog: A manually labelled multi-turn dialogue dataset. In IJCNLP, 986-995.

Serban, I. V.; Sordoni, A.; Bengio, Y.; Courville, A. C.; and Pineau, J. 2016. Building end-to-end dialogue systems using generative hierarchical neural network models. In AAAI, 3776-3784.

Xing, C.; Wu, W.; Wu, Y.; Liu, J.; Huang, Y.; Zhou, M.; and Ma, W. 2017. Topic aware neural response generation. In AAAI, 33513357.

Young, T.; Cambria, E.; Chaturvedi, I.; Zhou, H.; Biswas, S.; and Huang, M. 2018. Augmenting end-to-end dialogue systems with commonsense knowledge. In AAAI, 4970-4977.

Zhao, T.; Zhao, R.; and Eskénazi, M. 2017. Learning discourselevel diversity for neural dialog models using conditional variational autoencoders. In $A C L, 654-664$.

\footnotetext{
${ }^{1}$ https://www.opensubtitles.org
} 